ESAIM: PROCEEDINGS, March 2013, Vol. 39, p. 40-48

M. Belhaq, P. Lafitte and T. Lelièvre Editors

\title{
CHAOS AND SYNCHRONIZATION IN DELAYED SYSTEM : APPLICATIONS TO LASER NETWORKS
}

\author{
VALENTIN FLUNKERT ${ }^{1}$ AND ECKEHARD SCHÖLL ${ }^{2}$
}

\begin{abstract}
We discuss recent results on chaos synchronization of delayed systems. We investigate the limit of large coupling delays and discuss how in this limit the stability problem for the synchronized solution is drastically simplified. We use these results to derive rigorous conditions for chaos synchronization of all-optically coupled laser networks. In laser systems the optical coupling phases have to be taken into account and give rise to interference conditions. We show that the relative phases between lasers can be used to optimize the effective coupling matrix.
\end{abstract}

\section{INTRODUCTION}

Chaos synchronization has been a central topic of research in nonlinear sciences, due to its potential applications in secure communication. For technological chaos based applications, semi-conductor lasers provide a promising platforms, because they allow high bitrates and could exploit existing telecommunication infrastructure [3].

However, due to the fast dynamics of the lasers, propagation distances of already a few meters introduce non-negligible delay times in the coupling. The synchronization of delay-coupled systems in general and in particular delay-coupled lasers has thus been a focus of research in the last decades.

When the lasers are coupled all-optically, not only delay effects are important, but also the optical coupling phases of the coherently coupled electric fields play an important role. Coherent coupling may result in constructive or destructive interference of incoming signals.

The aim of this contribution is to derive synchronization conditions for all-optically coupled laser networks $[4,5]$. For our stability analysis we employ recent results concerning the master stability function (MSF) [9] in the limit of large delay times $[6,7]$. Furthermore, we discuss the role of the optical coupling phases and possible phase-shifts in the relative optical phases of the lasers.

The paper is organized as follows : In section 2 we review previous results from [6] (for more details please refer to this work). Our main results are stated in sections 3 and 4, where we apply these results to two mutually coupled lasers (section 3) and to networks of laser systems (section 4).

\footnotetext{
${ }^{1}$ Instituto de Fisica Interdisciplinar y Sistemas Complejos, IFISC (UIB-CSIC), Campus Universitat de les Illes Balears, E-07122 Palma de Mallorca, Spain 


\section{SynChronization of DELAY-COUPLED SYSTEMS}

Consider a delay-coupled network of $N$ identical units

$$
\dot{x}_{k}(t)=f\left(x_{k}(t)\right)+\sum_{j=1}^{N} G_{k j} h\left(x_{j}(t-\tau)\right),
$$

where $x_{k} \in \mathbb{R}^{n}$ is the state vector of the $k$-th node $(k=1, \ldots, N)$. Here, $f$ is a function describing the local dynamics of an isolated node, $G_{k j}$ is a coupling matrix that determines the coupling topology and the strength of each link in the network, $h$ is a coupling function, and $\tau$ is the delay time in the connection, which is assumed to be equal for all links. A necessary condition for synchronization is that the matrix $G$ has a constant row sum

$$
\text { C1 : } \quad \sigma=\sum_{j=1}^{N} G_{k j},
$$

independent of $k$. This condition ensures that an invariant synchronization manifold exists : Assume that all nodes start with the same history $\psi(t)=x_{k}(t)$ for $t \in[-\tau, 0]$. Then at $t=0$ the right hand side of Eq. (1) is independent of $k$ only if $\mathrm{C} 1$ holds.

Note that if $\mathrm{C} 1$ is fulfilled, the row sum $\sigma$ is an eigenvalue of the matrix $G$ corresponding to the eigenvector $(1,1, \ldots, 1)$ of synchronized dynamics. For a given constant row sum $\sigma$ the dynamics in the synchronization manifold of Eq. (1) is

$$
\dot{\bar{x}}(t)=f[\bar{x}(t)]+\sigma h[\bar{x}(t-\tau)] .
$$

where $\bar{x}(t)=x_{1}(t)=x_{1}(t)=\cdots=x_{N}(t)$ denotes the synchronized solution.

If condition $\mathrm{C} 1$ is satisfied, that is if a synchronization manifold exists, the stability problem of the synchronized solution can be approached using the MSF [9]. The MSF depends on a complex parameter $r e^{i \psi}$ and is defined as the largest Lyapunov exponent $\lambda\left(r e^{i \psi}\right)$ arising from the variational equation

$$
\dot{\xi}(t)=D f[\bar{x}(t)] \xi(t)+r e^{i \psi} D h[\bar{x}(t-\tau)] \xi(t-\tau),
$$

where $\bar{x}(t)$ is the synchronized trajectory of the system determined by Eq. (3) and $D f$ and $D h$ are Jacobians.

The synchronized state is stable for a given coupling topology if the MSF is negative at all transversal eigenvalues $\gamma_{k}$ of the coupling matrix $\left(\lambda\left(\gamma_{k}\right)<0\right)$. Here, transversal eigenvalue refers to all eigenvalues except for the eigenvalue $\sigma$ associated to perturbations within the synchronization manifold with corresponding eigenvector $(1,1, \ldots, 1)$.

When the coupling delay is larger than the dynamical time-scale of the nodes, as is the case for lasers coupled over distances of meters, the problem of calculating the master stability function is drastically simplified [6]. In this limit $(\tau \rightarrow \infty)$ it holds that

- The MSF is rotationally symmetric around the origin in the complex plane, i.e., $\lambda\left(r e^{i \psi}\right)$ is independent of $\psi$.

- If $\lambda(0)>0$, then $\lambda\left(r e^{i \psi}\right)=\lambda(0)$ for all $r$ (and $\psi$ ).

- If $\lambda(0)<0$, then the MSF is monotonically growing with respect to the parameter $r$ and there is a critical radius $r_{0}$, where it changes $\operatorname{sign}\left(\lambda\left(r_{0}\right)=0\right)$.

We briefly sketch the derivation of these results. For simplicity we use delayed maps. Consider first the simplest case of a fixed point in the synchronization manifold. In this case the variational equation (4) for the master stability function is (in the case of a map) given by

$$
\xi_{k+1}=A \xi_{k}+r e^{i \psi} B \xi_{k-\tau},
$$

where $A$ and $B$ are Jacobian matrices. The characteristic equation for the eigenvalues $z$ is given by

$$
\operatorname{det}\left[A-z I+r e^{i \psi} B z^{-\tau}\right]=0,
$$


where $I$ is the identity matrix. For large delay there are two types of solutions [11]. The strongly unstable solutions are solutions of the equation $\operatorname{det}[A-z I]=0$ with $|z|>1$, because such a value of $z$ is also a solution of Eq. (6) for $\tau \rightarrow \infty$. If such a solution exists, the fixed point is always transversely unstable.

The other type of solution is given by the pseudo-continuous spectrum. Inserting the ansatz $z=(1+\delta / \tau) e^{i \omega}$ into Eq. (4) and taking the limit $\tau \rightarrow \infty$, we obtain

$$
\operatorname{det}\left[A-I e^{i \omega}+r e^{i(\psi-\phi)} e^{-\delta} B\right]=0
$$

with $\phi=\omega \tau$. The parameter $\phi$ as well as $\omega$ take on arbitrarily dense values in $[-\pi, \pi]$ (the spacing of the solutions $\omega_{i}$ is given by $\left.\Delta \omega \approx 2 \pi / \tau\right)$. From this it is clear that the phase $\psi$ is unimportant for large delay, which explains the rotational symmetry. Furthermore, Eq. (7) can be solved for $\mu=r e^{-\delta} e^{i(\psi-\phi)}$. Then the branches $\delta(\omega)$ are given by $\delta(\omega)=-\ln |\mu(\omega)|+\ln r$. If the MSF is negative for $r=0$, it increases monotonically with increasing $r$ and it changes sign at some value $r_{0}$. Similar arguments can be given for periodic orbits and for chaotic attractors [6].

This structure of the MSF allows us to draw general conclusions about the synchronizability of a given network topology. In particular, chaos synchronization can only be stable if $\sigma$ is the eigenvalue of $G$ with largest magnitude, i.e., the transversal eigenvalues $\gamma_{1}, \gamma_{2}, \ldots \gamma_{N-1}$ have smaller magnitude

$$
\text { C2: } \quad\left|\gamma_{n}\right|<|\sigma| \text {. }
$$

This condition (C2) is necessary for chaos synchronization $\left(|\sigma|>r_{0}\right)$ and it is sufficient for synchronization on a periodic orbit $\left(|\sigma|<r_{0}\right)[6]$. In fact the smaller the magnitude of the transversal eigenvalues, the easier it is to synchronize the system.

For given synchronized dynamics (Eq. (3)), i.e., given system parameters and row sum $\sigma$, one can calculate numerically the critical radius $r_{0}$, which then provides a necessary and sufficient condition (C3) for synchronization (provided $\mathrm{C} 1$ is fulfilled)

$$
\text { C3 : } \quad\left|\gamma_{n}\right|<r_{0} .
$$

While condition C3 is necessary and sufficient, its disadvantage is that one needs to calculate $r_{0}$ explicitly for the particular synchronized dynamics. In contrast, condition C2 is only necessary for chaotic synchronization, however, its strengths lies in the fact that it depends solely on the coupling topology, i.e, the eigenvalues of $G$, and not on the particular dynamics of the system.

\section{Two LASERS}

In this section we consider two semiconductor lasers that are delay-coupled to each other with a coupling delay and additionally receive self-feedback with the same delay time $\tau$. The coupled system is described by dimensionless rate equations of Lang-Kobayashi type [8]

$$
\begin{aligned}
\dot{E}_{1} & =\frac{1}{2}(1+i \alpha)\left[G\left(n_{1}, E_{1}\right)-1\right] E_{1}+\kappa_{11} e^{i \phi_{11}} E_{1}(t-\tau)+\kappa_{12} e^{i \phi_{12}} E_{2}(t-\tau), \\
\dot{E}_{2} & =\frac{1}{2}(1+i \alpha)\left[G\left(n_{2}, E_{2}\right)-1\right] E_{2}+\kappa_{22} e^{i \phi_{22}} E_{2}(t-\tau)+\kappa_{21} e^{i \phi_{21}} E_{1}(t-\tau), \\
T \dot{n}_{1} & =p-n_{1}-G\left(n_{1}, E_{1}\right)\left|E_{1}\right|^{2}, \\
T \dot{n}_{2} & =p-n_{2}-G\left(n_{2}, E_{2}\right)\left|E_{2}\right|^{2},
\end{aligned}
$$

where $E_{k}$ and $n_{k}$ are the normalized complex electric field amplitude and the rescaled inversion of the $k$-th laser, respectively, and $\alpha$ is the linewidth enhancement factor, $p$ is the normalized pump current in excess of the threshold, and $\kappa_{i j}, \phi_{i j}$ are the coupling amplitudes and phases, respectively. The gain is modeled by $G(n, E)=(n+1) /\left(1+\mu|E|^{2}\right)$, where the denominator takes into account gain saturation effects. Throughout this paper we choose the following model parameters for our numerical simulations, unless stated otherwise : Ratio between carrier and photon lifetime $T=1000, p=0.1, \alpha=4$, gain saturation $\mu=0.26$. 
The optical coupling phases $\phi_{i j}$ are determined by the optical path lengths of the feedback and coupling sections on a subwavelength scale $\phi_{i j}=\Omega_{0} \tau_{i j}$, where $\Omega_{0}$ is the optical frequency of the laser. Since $\Omega_{0}$ is very $\operatorname{large}^{1}$, one can consider the phases as parameters independent of the coupling delays. We thus choose all delays equal, but consider the phases as free parameters.

One important peculiarity of coupled lasers is that the lasers may synchronize with a relative phase shift $u$, i.e., $E_{1}(t)=e^{i u} E_{2}(t)$. To explicitly treat this relative phase shift, we transform to the new variable $\tilde{E}_{2}$ as $\tilde{E}_{2}(t)=e^{i u} E_{2}(t)$. After substituting this into Eqs. (10) and omitting the tildas for simplicity we arrive at the following rate equations for the fields

$$
\begin{aligned}
& \dot{E}_{1}=\ldots+\kappa_{11} e^{i \phi_{11}} E_{1}(t-\tau)+\kappa_{12} e^{i\left(\phi_{12}-u\right)} E_{2}(t-\tau) \\
& \dot{E}_{2}=\ldots+\kappa_{22} e^{i \phi_{22}} E_{2}(t-\tau)+\kappa_{21} e^{i\left(\phi_{21}+u\right)} E_{1}(t-\tau)
\end{aligned}
$$

The artificially introduced parameter $u$ helps in the analysis of synchronization, because we can discuss existence and stability of the synchronized solution $E_{1}(t)=E_{2}(t)$ in dependence on $u$, thereby treating synchronization of the lasers with a phase shift.

Bringing Eq. (11) into the form of Eq. (1) essentially yields the coupling matrix

$$
G=\left[\begin{array}{ll}
\kappa_{11} e^{i \phi_{11}} & \kappa_{12} e^{i\left(\phi_{12}-u\right)} \\
\kappa_{21} e^{i\left(\phi_{21}+u\right)} & \kappa_{22} e^{i \phi_{22}}
\end{array}\right]
$$

The row sum condition $\mathrm{C} 1$ is then given by

$$
\kappa_{11} e^{i \phi_{11}}+\kappa_{12} e^{i\left(\phi_{12}-u\right)}=\kappa_{22} e^{i \phi_{22}}+\kappa_{21} e^{i\left(\phi_{21}+u\right)} .
$$

Equation (13) can be interpreted as follows : If for a given set of coupling strengths and coupling phases there exists a phase shift $u$, such that Eq. (13) is fulfilled, then there exists an invariant synchronization manifold. The lasers can thus tune their relative phase shifts appropriately. The stability of the synchronized solution then determines whether synchronization will be observable or not.

As a starting point for the stability analysis we consider the MSF for a laser network with row sum $\sigma$. Since we focus on the large delay case $\left(\tau=1000 \gg T_{R O}\right.$, where $T_{R O}$ is the intrinsic relaxation oscillations period) the rotational symmetry discussed above holds and the MSF $\lambda\left(r e^{i \psi}\right)$ depends solely on $r$. Figure 1(a) and (b) depicts the MSF in the $(\sigma, r)$-plane for two different values of the pump current $p=0.1$ (panel (a)) and $p=1$ (panel (b)). When the critical radius $r_{0}$ (solid line) lies below the diagonal line $r=\sigma$ (dotted line), the synchronized solution is chaotic for a network with this row sum, since $\lambda(\sigma)>\lambda\left(r_{0}\right)=0$. When $r_{0}=\sigma$, as occurs for instance in panel (b) for small values of $\sigma$, the synchronized dynamics is periodic. In this case the solution $\lambda=0$ corresponds to the Goldstone mode of the periodic orbit.

In the following we will consider different coupling topologies of the two lasers. Figure 1(c) depicts all possible network motifs (up to exchange of $1 \leftrightarrow 2$ ) with more than one connection. The motifs on the left (i-iv) can exhibit chaos synchronization in the limit of large delays, while those on the right (v-vii) cannot [6] (trivially for motif (vii)). We will illustrate the analysis for motif (ii). For the other motifs, the analysis is similar, albeit it might be more involved [5].

We will now discuss the implications of the synchronization conditions C1-C3 for these motifs and compare the predictions with numerical simulations. We study two measures for synchronization : The correlation coefficient $\rho$ of the laser intensities $I_{1}, I_{2}$ and the synchronization probability $P_{S}$

$$
\rho=\frac{\left\langle\left(I_{1}-\left\langle I_{1}\right\rangle\right)\left(I_{2}-\left\langle I_{2}\right\rangle\right)\right\rangle}{\sqrt{\left(\Delta I_{1}\right)^{2}\left(\Delta I_{2}\right)^{2}}}, \quad P_{S}=\operatorname{Prob}\left(\frac{\left|I_{1}(t)-I_{2}(t)\right|}{\left\langle I_{1}\right\rangle+\left\langle I_{2}\right\rangle}<\varepsilon\right) .
$$

\footnotetext{
${ }^{1}$ Typically, the optical frequency is of the order $10^{14} \mathrm{~s}^{-1}$, corresponding to a value of $\Omega_{0} \approx 10^{24}$ in the dimensionless units used here.
} 

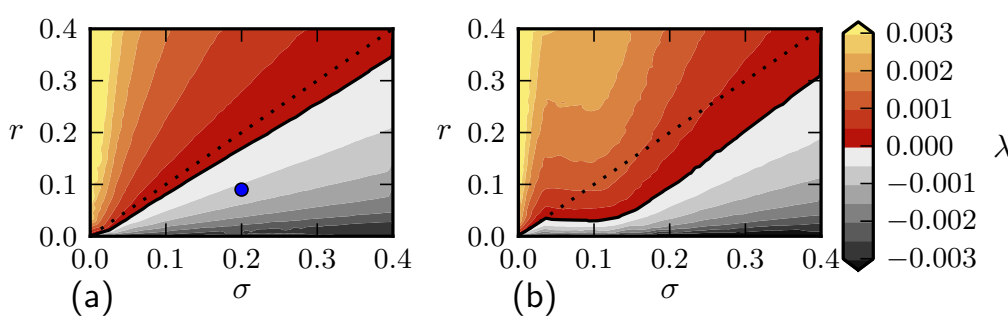

(b)

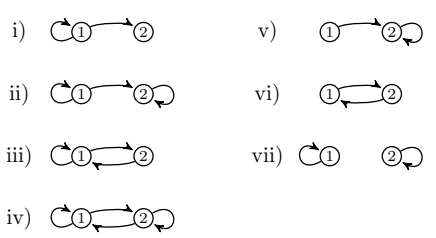

(c)

Figure 1. Panel (a) and (b) : MSF $\lambda(r)$ in the $(\sigma, r)$-plane for $p=0.1$ (panel (a)) and $p=1.0$ (panel (b)). The solid line depicts the critical radius $r_{0}$ with $\lambda\left(r_{0}\right)=0$. The dotted line is the diagonal line $r=\sigma$. The blue dot corresponds to a parameter set that we will use in the following numerical investigations.

Panel (c) : Possible motifs for two delay-coupled lasers. Motifs i-iv can (for certain parameters) exhibit zero-lag chaos synchronization and are discussed in more detail below. The motifs v-vii cannot exhibit zero-lag chaos synchronization.

Here, $\langle\cdot\rangle$ denotes the time average and $\left(\Delta I_{k}\right)^{2}$ denotes the variance of the respective intensity. Although, the correlation coefficient can in principle distinguish between identical synchronization $(\rho=1)$ and imperfect (generalized) correlation $(\rho<1)$, it is not the most sensitive measure for this purpose, because imperfect synchronization may still yield very large correlation $\rho \approx 1$.

To overcome this disadvantage of the correlation coefficient, we calculate the synchronization probability $P_{S}$. $P_{S}$ is defined as the probability that at any time $t$ the relative error between $I_{1}(t)$ and $I_{2}(t)$ is smaller than a threshold $\varepsilon$ We choose $\varepsilon=0.01$ in the following.

We now show how the conditions C1-C3 can be discussed for a laser network. We focus on the motif (ii) in Fig. 1c. This setup consists of two unidirectionally coupled lasers with self-feedback and has been studied in different contexts. The importance of the coupling phases in this coupling scheme has been recognized in [10]. In this reference it was observed in an experiment that depending on the (relative) feedback phases the synchronization behavior ranges from perfect synchronization to an almost uncorrelated state. So far these experiments have not been sufficiently explained. It turns out that the experimental results can be well understood in the light of the synchronization conditions $\mathrm{C} 1-\mathrm{C} 3$ :

The coupling matrix corresponding to motif (b) is given by

$$
G=\left[\begin{array}{ll}
\kappa_{11} e^{i \phi_{11}} & 0 \\
\kappa_{21} e^{i\left(\phi_{21}+u\right)} & \kappa_{22} e^{i \phi_{22}}
\end{array}\right]
$$

and the row sum condition $\mathrm{C} 1$ can be brought into the form

$$
\kappa_{21}=\left|\kappa_{11}-\kappa_{22} e^{i \Phi_{\text {rel }}}\right|=\sqrt{\kappa_{11}^{2}+\kappa_{22}^{2}-2 \kappa_{11} \kappa_{22} \cos \left(\Phi_{\text {rel }}\right)}
$$

with $\Phi_{\text {rel }}=\phi_{22}-\phi_{11}$, by eliminating the relative phase $u$. This phase shift $u$ between the laser can then be calculated and is given by $u=\phi_{11}-\phi_{21}+\operatorname{Arg}\left(\kappa_{11}-\kappa_{22} e^{i \Phi_{\text {rel }}}\right)$ where Arg denotes the complex argument.

Condition $\mathrm{C} 2$ on the other hand reads for this case $\kappa_{22}<\kappa_{11}=|\sigma|$, i.e., chaos synchronization is possible, if the self-coupling of laser 2 is weaker than that of laser 1 . For a given set of parameters, synchronization is stable if condition C3 is fulfilled, i.e., if $\kappa_{22}<r_{0}\left(\kappa_{11}\right)$.

To illustrate these conditions, we consider the following parameter set

$$
\kappa_{11}=0.2, \quad \kappa_{22}=0.09, \quad \phi_{11}=0, \quad \phi_{21}=0
$$

and vary $\Phi_{\text {rel }}=\phi_{22}$ and $\kappa_{21}$. These coupling strengths $(\sigma, r)=\left(\kappa_{11}, \kappa_{22}\right)$ are marked by a blue dot in Fig. 1a and are chosen such that synchronization is stable (condition C3 is fulfilled). Figure 2 depicts the correlation 

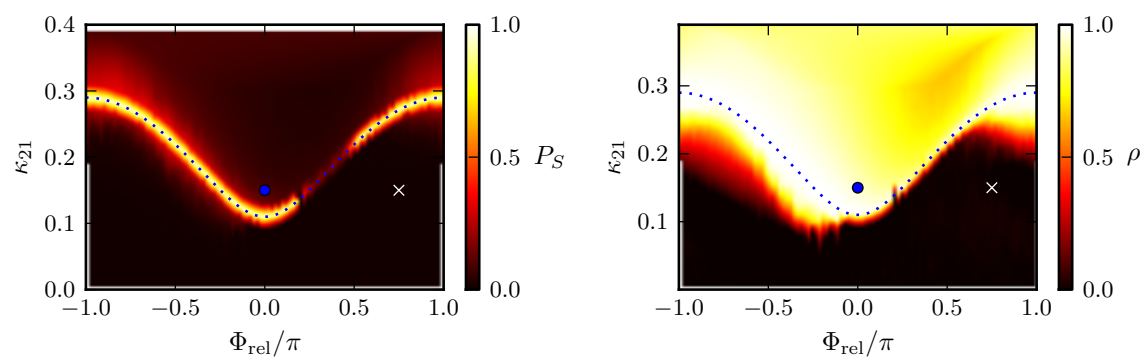

Figure 2. (Motif (b)) Synchronization probability $P_{S}$ (left) and correlation coefficient $\rho$ (right) as a function of $\phi_{22}=\Phi_{\text {rel }}$ and $\kappa_{21}$. The dotted line corresponds to the synchronization condition C1 (Eq. (16)). Other parameters : $\kappa_{11}=0.2, \kappa_{22}=0.09, \phi_{11}=\phi_{21}=0$.

coefficient $\rho$ and the synchronization probability $P_{S}$ in the $\left(\Phi_{\mathrm{rel}}, \kappa_{21}\right)$-plane. The dotted line corresponds to Eq. (16), where condition $\mathrm{C} 1$ is satisfied. This condition clearly coincides with high synchronization probabilities.

As discussed above, high correlations are also possible without perfect synchronization. In the regions of high correlation and low synchronization probabilities, we observe generalized synchronization.

The blue dotted line (condition C1) in Fig 2 roughly marks the boundary between regions of high and low correlations. Below the line, we observe no synchronization at all and the correlation in this case is very low.

In Fig. 2(left) there is a region on the dotted line $\Phi_{\text {rel }} \in[0.2 \pi, 0.5 \pi]$, where the synchronization probability is low. In this region we observe multistability between identical synchronization solution, a state of generalized synchronization. Which state is chosen depends in our deterministic simulations sensitively on the initial conditions. Including noise in the simulation results in spontaneous switching between the three states, albeit it is hard to distinguish between identical synchronization and generalized synchronization in the presence of noise.

Thus although our synchronization condition C1-C3 give the correct existence and stability of the identical synchronized solution, we certainly cannot exclude the existence of other attractors.

The experimental investigations in Ref. [10] were performed under similar parameter conditions, i.e., $\kappa_{22}<\kappa_{11}$ (obeying condition C2). As $\Phi_{\text {rel }}$ was varied, the correlation varied from almost perfect to almost no correlation. Varying $\Phi_{\text {rel }}$ for a fixed value of $\kappa_{21}$ in Fig. 2 reproduces this behavior, provided the value of $\kappa_{21}$ intersects the dotted synchronization curve. Thus the experimental results can be understood as interference effects, which may or may not lead to the existence of a synchronization manifold, corresponding to high and low correlation, respectively. The other motifs shown in Fig. 1c, can be discussed in a similar way [5].

\section{LASER NETWORKS}

We now move from the two-laser system to networks of $N$ all-optically coupled lasers. The synchronization of laser networks is on one hand important for applications for instance in high power laser arrays, where the synchronization of optical phases yields an intensity $I \propto N^{2}$ for interfering beams in contrast to the case of randomly distributed phases that gives $I \propto N$.

On the other hand, laser networks have been proposed as optical information processing systems $[1,2]$. Understanding stability properties of dynamical states in these networks is a necessary first step for controlling and utilizing these systems.

For a network of $N$ all-optically coupled lasers, the coupling terms in the Lang-Kobayashi rate equations are given by

$$
\dot{E}_{k}=\cdots+\sum_{j=1}^{N} \kappa_{k j} e^{i \phi_{k j}} E_{j}(t-\tau) .
$$

Here $\kappa_{k j}$ and $\phi_{k j}$ are matrices describing the coupling strength and coupling phase, respectively, of the connection $j \rightarrow k$. Again the lasers may synchronize with relative phase shifts $u_{k}$, which we define with respect to laser 


$$
G=\left[\begin{array}{ccccc}
0 & G_{12} & & & G_{1 N} \\
G_{21} & 0 & G_{23} & & \\
& G_{32} & \ddots & \ddots & \\
& & \ddots & \ddots & G_{N-1, N} \\
G_{N 1} & & & G_{N, N-1} & 0
\end{array}\right]
$$

(a)

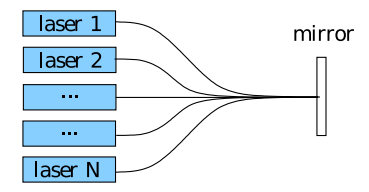

(b)

Figure 3. Panel (a) : Principal structure of coupling matrix for a bidirectional ring. The matrix entries are given by $G_{k j}=\kappa_{k j} e^{i\left(\phi_{k j}+u_{k}-u_{j}\right)}$ and depend on the relative phases $u_{k}$. Panel (b) : All-to-all coupling of multiple lasers via a common mirror.

one $E_{1}(t)=e^{i u_{k}} E_{k}(t)$, i.e., $u_{1}=0$. Performing the corresponding transformation (as before in Eq. (11)) $\tilde{E}_{k}(t)=e^{i u_{k}} E_{k}(t)$ and omitting the tildas for simplicity we obtain the field equations

$$
\dot{E}_{k}=\cdots+\sum_{j=1}^{N} \kappa_{k j} e^{i\left(\phi_{k j}+u_{k}-u_{j}\right)} E_{j}(t-\tau) .
$$

The necessary condition $\mathrm{C} 1$ for synchronization is then stated as follows : There exists an invariant synchronization manifold if and only if there is a combination of relative phases $u_{k}(k=2, \ldots, N)$, such that the complex row sum

$$
\sigma_{k}=\sum_{j=1}^{N} \kappa_{k j} e^{i\left(\phi_{k j}+u_{k}-u_{j}\right)}
$$

is independent of $k$. As we saw before, this condition is already very difficult to analyze for two lasers when all four connections are present and in an experiment it is virtually impossible to control more than a few phases. For larger networks, optoelectronic, incoherent optical feedback or coupling via a common relay may thus be a more promising coupling method. However, in certain cases all-optical coupling may be feasible in an experimental situation.

To illustrate the complexity of the synchronization condition $\mathrm{C} 1$ in a simple case, we consider a system of bidirectionally coupled lasers.

\subsection{Rings of bi-directionally coupled lasers}

For rings of bi-directionally coupled lasers, the coupling matrix has the principal structure shown in Fig. 3a with $G_{k j}=\kappa_{k j} e^{i\left(\phi_{k j}+u_{k}-u_{j}\right)}$. For this network matrix we obtain the $N$ complex row sums $\sigma_{k}$ and the necessary synchronization condition $\mathrm{C} 1$ corresponds to $2(N-1)$ equations (each complex equation yields two real equations)

$$
\sigma_{1}=\sigma_{2}, \quad \sigma_{2}=\sigma_{3}, \quad \ldots \quad \sigma_{N-1}=\sigma_{N} .
$$

However, there are only $N-1$ relative phases $u_{j}\left(u_{1}=0\right)$ that the system can choose freely. Thus, in an experiment we need to control $N-1$ coupling phases to satisfy the synchronization condition C1. For a ring of $N=3$ elements, controlling two coupling phases may still be feasible, but this approach quickly fails for larger $N$.

\subsection{Coupling via a common mirror}

We will now discuss one promising all-optical setup that should, in principle, be robust to phase mismatches. Consider the setup [1] sketched in Fig. 3b. The laser fields are coupled into a common fiber, which is terminated by a mirror. As before, we assume that the coupling delays are equal (the light paths are equally long on a $\mathrm{cm}$ scale), but allow for coupling phases, i.e., differences in optical path-lengths on wavelength scales. In this setup, the connection from each laser $k$ to the mirror corresponds to a certain optical path length with a corresponding phase $\psi_{k}$. Similarly, the connection from each laser to the mirror has an associated coupling strength $c_{k}$, which could in an experiment be controlled by an attenuator in the corresponding fiber. The coupling phase $\phi_{k j}$ and 
the coupling strength $\kappa_{k j}$ from laser $j$ to laser $k$ are then given by

$$
\phi_{k j}=\psi_{k}+\psi_{j} \quad \text { and } \quad \kappa_{k j}=c_{k} \cdot c_{j}
$$

Under these conditions, the row sum is given by

$$
\sigma_{k}=\sum_{j=1}^{N} \kappa_{k j} e^{i\left(\phi_{k j}+u_{k}-u_{j}\right)}=c_{k} e^{i\left(\psi_{k}+u_{k}\right)} \sum_{j=1}^{N} c_{j} e^{i\left(\psi_{j}-u_{j}\right)} .
$$

The sum on the right hand side is a complex number independent of $k$. The row sum condition can thus be satisfied if the prefactor is also independent of $k$, i.e., all coupling strengths are equal $\left(c_{k}=c\right)$ and the relative phases $u_{k}$ are chosen by the system to give ${ }^{2} \psi_{1}=\psi_{k}+u_{k}$ with $k=2, \ldots, N$. In essence, all lasers couple to the optical mean field and compensate for the difference in optical path length of their individual fiber by adjusting their relative phase shift. As long as the setup obeys Eqs. (22), the coupling phases $\psi_{k}$ may vary, e.g., due to thermal effects, and do not need to be controlled.

Assuming that all coupling strengths are equal and the relative phases $u_{k}$ are tuned appropriately by the system the coupling matrix is given by

$$
G_{k j}=c^{2} e^{i\left(\psi_{k}+\psi_{j}+u_{k}-u_{j}\right)}=c^{2} e^{i 2 \psi_{j}}
$$

This matrix has one eigenvalue corresponding to the row sum $\sigma=c^{2} \sum_{j=1}^{N} e^{i 2 \psi_{j}}$ and the $N-1$ transversal eigenvalues are zero $\left(\gamma_{n}=0\right.$ with $\left.n=1, \ldots, N-1\right)$, such that this setup is optimal for synchronization.

Recently, this coupling scheme has been proposed for optical information processing using multi-mode lasers [1]. For multi-mode lasers, the same argument as above holds for each mode, such that the setup is, in principle, robust to phase mismatches. However, it may still be difficult to realize the assumptions Eqs. (22) in an experimental setup.

\section{Conclusion And outlook}

We have discussed chaos synchronization conditions for all-optically coupled lasers. In all-optical coupling, the coupling phases play a crucial role for the synchronizability. The condition of constant row sum corresponds to specific interference conditions, i.e., the input signals of each laser should interfere in such a way that each laser receives the same input signal, relative to its own phase. This corresponds to the existence of an identical synchronization manifold. Through interference, the phases may compensate for mismatches in the coupling strengths.

Using these interference arguments we have explained experimental findings [10] and discussed necessary and sufficient conditions for synchronization of a network motif of two all-optically coupled lasers.

Further, we have considered synchronization of larger laser networks, and singled out the difficulties that arise in all-optical coupling schemes due to the interference conditions. We predict that a setup of all-to-all coupling via a common mirror may under certain conditions be robust to phase mismatches and thus be optimal with respect to stability of the synchronized chaotic dynamics.

Acknowledgement — We thank Andreas Amann, Ingo Fischer, and Serhiy Yanchuk for helpful discussions. This work was supported by DFG in the framework of Sfb 910. VF acknowledges financial support by the German Academic Exchange Service (DAAD).

\footnotetext{
${ }^{2}$ The special role of laser 1 stems from our choice $u_{1}=0$.
} 


\section{REFERENCES}

[1] A. Amann, A. Pokrovskiy, S. Osborne, and S. O'Brien. Complex networks based on discrete-mode lasers. In International Workshop on Multi-Rate Processes and Hysteresis, Cork 2008, volume 138 of J. Phys. : Conf. Ser., page 012001. IOP Publishing, 2008.

[2] L. Appeltant, M. C. Soriano, Guy Van der Sande, J. Danckaert, S. Massar, J. Dambre, B. Schrauwen, C. R. Mirasso, and I. Fischer. Information processing using a single dynamical node as complex system. Nat. Commun., 2 :468, 2011.

[3] A. Argyris, D. Syvridis, L. Larger, V. Annovazzi-Lodi, P. Colet, I. Fischer, J. García-Ojalvo, C. R. Mirasso, L. Pesquera, and K. A. Shore. Chaos-based communications at high bit rates using commercial fibre-optic links. Nature, 438 :343-346, 2005.

[4] V. Flunkert. Delay-Coupled Complex Systems. Springer Theses. Springer, Heidelberg, 2011.

[5] V. Flunkert and E. Schöll. Chaos synchronization in networks of delay-coupled lasers : Role of the coupling phases. New. J. Phys., 14 :033039, 2012.

[6] V. Flunkert, S. Yanchuk, T. Dahms, and E. Schöll. Synchronizing distant nodes : a universal classification of networks. Phys. Rev. Lett., $105: 254101,2010$.

[7] S. Heiligenthal, T. Dahms, S. Yanchuk, Thomas Jüngling, V. Flunkert, I. Kanter, E. Schöll, and W. Kinzel. Strong and weak chaos in nonlinear networks with time-delayed couplings. Phys. Rev. Lett., $107: 234102,2011$.

[8] R. Lang and K. Kobayashi. External optical feedback effects on semiconductor injection laser properties. IEEE J. Quantum Electron., $16: 347-355,1980$.

[9] L. M. Pecora and T. L. Carroll. Master stability functions for synchronized coupled systems. Phys. Rev. Lett., 80(10) :21092112, 1998

[10] M. Peil, T. Heil, I. Fischer, and W. Elsäßer. Synchronization of chaotic semiconductor laser systems : A vectorial couplingdependent scenario. Phys. Rev. Lett., 88(17):174101, 2002.

[11] S. Yanchuk and P. Perlikowski. Delay and periodicity. Phys. Rev. E, 79(4):046221, 2009. 